\title{
Panoramas de la alfabetización académica en el ámbito iberoamericano: Aportes para la calidad de la Educación Superior
}

Panoramic views of academic literacy in the Ibero-American sphere: contributions to the quality of Higher Education

Juan Antonio Núñez Cortés

e-mail: juanantonio.nunnez@uam.es

Universidad Autónoma de Madrid. España

\author{
María Constanza Errázuriz Cruz \\ e-mail: cerrazuc@uc.cl \\ Pontificia Universidad Católica de Chile. Chile
}

\section{Resumen}

El desarrollo de la alfabetización académica ha sido objeto de interés en el ámbito iberoaméricano en las últimas décadas. De este interés se han derivado multitud de investigaciones sobre la enseñanza de la lectura y la escritura en la universidad e iniciativas que han tenido como objetivo paliar las dificultades en la competencia comunicativa de los estudiantes en el nivel superior así como hacer que estos se familiaricen con las culturas discursivas de las disciplinas en las que se forman. El propósito de este monográfico es difundir investigaciones y propuestas sobre alfabetización académica en el mundo hispano, con el fin de contribuir a comprender el problema más profundamente y desarrollar esta línea de investigación para afianzar y elevar la calidad de los dispositivos de escritura académica y, de este modo, los programas de formación de la educación superior. A partir de este marco de referencia, cada artículo del monográfico aborda distintos enfoques y factores que intervienen en el tratamiento de la lectura y escritura académicas en diversos contextos, junto con resultados de dispositivos y programas de escritura académica.

Palabras clave: alfabetización académica; educación superior; equidad; lectura; escritura.

\begin{abstract}
The development of academic literacy has been an object of interest in the Ibero-American sphere in recent decades. From this interest, a multitude of investigations have been derived on the teaching of reading and writing at the university and initiatives that have aimed to alleviate the difficulties in the communicative competence of students at the higher level as well as making them familiar with the discursive cultures of the disciplines in which they are formed. The purpose of this monograph is to disseminate research and proposals on academic literacy in the Hispanic world, in order to help understand the problem more deeply and develop this line of research to strengthen and raise the quality of academic writing devices and, from Thus, higher education training programs. Based on this frame of reference, each article of the monograph addresses different approaches and factors that intervene in the treatment of academic reading and writing in various contexts, together with results from devices and academic writing programs.
\end{abstract}

Keywords: academic literacy; higher education; equity; reading; writing.

Recibido / Received: 02-06-2020

Aceptado / Accepted: 22-06-2020

Publicado / Published: 01-07-2020

Cómo referenciar este artículo / How to reference this article:

Núñez, J. A., \& Errázuriz, C. (2020). Panoramas de la alfabetización académica en el ámbito iberoamericano: aportes para la calidad de la Educación Superior. Tendencias Pedagógicas, 36, pp. 1-8. doi: 10.15366/tp2020.36.01 
¿Por qué los estudiantes que ingresan actualmente en las universidades presentan competencias insuficientes para leer y escribir textos académicos? Son muchas las respuestas que se pueden dar para explicar este fenómeno: la falta de calidad de la educación escolar, las nuevas tecnologías de la información y la comunicación y el incremento sostenido del número de estudiantes que pueden acceder a la educación superior, entre otras. Sin embargo, las reiteradas quejas escuchadas sobre este problema no ofrecen soluciones a una realidad que está presente y ciertamente se mantendrá. Está claro, entonces, que debemos hacernos cargo de esta situación, pero ¿cómo? Los docentes universitarios esperan que las escuelas e institutos se responsabilicen de esto; asimismo, los profesores de educación secundaria culpan a la educación primaria, pero de este modo el problema persiste. En consecuencia, son las instituciones de educación superior, las facultades y, específicamente, los docentes universitarios, quienes debemos tomar medidas para preparar a los estudiantes para el mundo académico y laboral.

Sabemos que el dominio de la lectura y la escritura son las metas más relevantes de la escuela, pero no solo de estas instituciones, sino también de las instituciones de educación superior. A diferencia de la creencia extendida, comprender y producir textos no es una habilidad que se logre de una vez y para siempre, sino que -como afirman Cassany (2006) y Carlino (2005) - son competencias que se desarrollan a lo largo de toda la vida y que se han de adaptar según el contexto. Es decir, demandan años de entrenamiento y de práctica en su uso en distintas situaciones comunicativas. En este sentido, las competencias comunicativas necesarias para desenvolverse en la universidad no son las mismas para la educación escolar. De hecho, aunque la educación primaria y secundaria tuviesen una gran calidad, aún así los jóvenes no llegarían totalmente preparados para enfrentar la educación superior ¿Por qué? Porque los textos que se leen y escriben en la universidad y en cada disciplina son propios de esos ámbitos y los estudiantes probablemente no han estado expuestos a ellos ni a esas situaciones de comunicación específicas y no han participado de esas comunidades. Incluso, cada área del conocimiento tiene sus propios géneros discursivos, códigos y lenguaje particulares (Parodi et al., 2010). Por lo tanto -como lo han señalado Bazerman et al. (2005, 2012), Carlino (2005; 2012), Castelló (2009) y Russell (1990), tanto en países como EE. UU. como en los europeos y latinoamericanos- las universidades deben preparar a los estudiantes para comprender y producir textos propios del ámbito académico a través del currículo de los programas de formación, de modo que sean capaces de enfrentarse con éxito al mundo laboral y poder actualizarse constantemente. Así, han surgido movimientos académicos como Writing Across the Curriculum (WAC) y Writing in disciplines (WID), como posibles soluciones a las dificultades de lectura y escritura de los estudiantes de educación superior.

Por otra parte, ¿cuál es la importancia de la lectura y escritura para áreas del conocimiento alejadas del estudio del lenguaje? Efectivamente, estas destrezas tienen una relevancia capital para todas las áreas del saber, pues son los medios y herramientas de aprendizaje ineludibles para acceder al conocimiento, para internalizarlo, transformarlo y, finalmente, producir uno nuevo (Bereiter \& Scardamalia, 1987; Carlino, 2005; Castelló, 2009). Ciertamente, esta es justamente una de las actividades propias del mundo académico: acceder al nuevo saber, modificarlo, construir un nuevo conocimiento, comunicarlo a través de artículos científicos y enseñarlo a los estudiantes; vale decir, enseñar lo que se investiga.

Lo anteriormente expuesto adopta especial relevancia al considerar que la educación superior en Iberoamérica -en la última década- ha experimentado un notable crecimiento, debido a sus políticas de equidad y democratización, posibilitando el ingreso de estudiantes de "primera generación". Sin embargo, esta apertura de las instituciones igualmente ha causado situaciones de inequidad por las dificultades de inserción de los estudiantes más desaventajados (Ezcurra, 2011; Santelices et al., 2018; Silveira, 2018). En efecto, para que la educación superior sea realmente inclusiva es necesario asegurar no solo el acceso, sino también la continuidad de los estudiantes en sus carreras y la calidad de estas (Ezcurra, 2011). Por ende, se han generado iniciativas que, por un lado, han dado respuesta a las barreras que imponen las prácticas de lectura y escritura propias de la cultura académica y, por el otro, facilitan la apropiación de los discursos disciplinares (Natale y Stagnaro, 2017). De hecho, algunos dispositivos de desarrollo de la comunicación académica han resultado ser exitosos para el incremento del desempeño de la escritura académica por parte de los estudiantes universitarios, ya que ha demostrado mejorar el rendimiento de sus participantes en cuanto a sus productos escritos y 
hacer progresar sus concepciones de escritura (Errázuriz, 2016, 2017; Martin \& Cervi, 2006; Thaiss et al., 2012), debido a que pueden facilitar la inserción y enculturación de los estudiantes a la cultura académica desde sus propias experiencias como escritores y estudiantes y el reforzamiento de su identidad como escritores (Ivanic, 1998; Natale \& Stagnaro, 2017). Asimismo, estos dispositivos pueden tener un alto potencial para elevar la calidad de la formación, como señala la evidencia internacional (Thaiss et al., 2012). No obstante, en algunas ocasiones este potencial no es aprovechado totalmente o no se ha medido aún, de ahí que sea un imperativo continuar investigando sobre los resultados de estas iniciativas.

En consecuencia, el propósito de este monográfico es difundir investigaciones y propuestas sobre alfabetización académica en el mundo hispano, con el fin de contribuir a comprender el problema más profundamente y desarrollar esta línea de investigación para afianzar y elevar la calidad de los dispositivos de escritura académica y, de este modo, los programas de formación de la educación superior. A partir de este marco de referencia, cada artículo del monográfico aborda distintos enfoques y factores que intervienen en el tratamiento de la lectura y escritura académicas en diversos contextos, junto con resultados de dispositivos y programas de escritura académica. Como es posible observar, el orden de los artículos en el monográfico presenta detrás una lógica, pues partimos con aquellos más teóricos y particulares, para luego avanzar a investigaciones más amplias y aplicadas.

Tal y como se ha indicado anteriormente, uno de los principales retos a los que se enfrentan los estudiantes universitarios es al hecho de tener que escribir géneros discursivos académicos con los que no están familiarizados. Así, uno de los que más dificultades genera es el llamado trabajo de fin de grado, tesis de grado, seminario o tesina, en función del país. De estas dificultades y de la enseñanza de este género en particular trata el artículo Los trabajos de fin de grado en español y en inglés. Retos, y un intento de mejora, de la alfabetización académica en formación inicial de profesorado realizado por Aoife Ahern y Alicia Hernando. Así, las autoras abordan los problemas que tienen los estudiantes a la hora de escribir un texto en el que se espera que muestren las competencias adquiridas durante la carrera, pero para el que no se les ha preparado.

Es cierto que, por ejemplo, en España este trabajo constituye una sola asignatura al final del grado universitario cuya dinámica consiste en la tutorización del trabajo por parte de un profesor de la universidad al que se le reconocen muy pocas horas de docencia por esta labor. Asimismo, este profesor, que puede ser experto o no en el tema escogido por el estudiante, si bien tiene experiencia docente e investigadora, quizá no tenga formación por ejemplo en estrategias de retroalimentación de textos escritos. Sea como fuere, con independencia de que el tutor/profesor esté más o menos formado y comprometido con su función de supervisión del trabajo, el estudiante se enfrenta a todo un reto académico que generalmente produce, cuando menos, preocupación. Quizá una de los motivos sea la ausencia de coherencia en la gradación de la enseñanza de los diferentes géneros discursivos durante la carrera y la falta de transversalidad en las actividades de escritura de las diferentes asignaturas cursadas en la universidad. De tal manera que los estudiantes ante la tarea, tal y como indican las autoras, se encuentra con dificultades como la incapacidad para integrar las voces ajenas y de dotar de coherencia y cohesión las ideas presentadas; el desconocimiento de algunas convenciones académicas; o la incertidumbre al encontrarse pautas de redacción insuficientes y contradictorias en las guías de escritura de este tipo de textos.

$\mathrm{Al}$ respecto, y para solventar estas dificultades, se llevan a cabo en las universidades diferentes iniciativas de alfabetización académica, algunas de las cuales se están exponiendo en esta introducción. Una de las más frecuentes son los talleres, a veces in extremis, de escritura del trabajo de fin de grado, al igual que otros como los de escritura de la tesis de maestría o trabajo de fin de máster, de tesis doctoral, de artículos de investigación o de ensayos. El objetivo de estos talleres es familiarizar al estudiante con el género discursivo objeto de estudio y así, generalmente, se presenta una conceptualización del género, su estructura y sus características lingüísticas, y se analizan y comentan modelos. Al respecto, basado en el enfoque de "Leer para aprender" de Rose y Martin (2012), Ahern y Hernando proponen en su trabajo un taller de escritura con base en una pedagogía de los géneros discursivos centrada en la lectura, análisis y redacción de textos. Asimismo, dan cuenta de la óptima percepción que tienen sobre el taller no solo los estudiantes que lo cursaron sino también sus tutores, al percibir en los primeros más autonomía para la redacción de sus trabajos.

Otra de las dificultades que se ha identificado es la escritura de la tesis por parte de estudiantes de postgrado, quienes, pese a ir cumpliendo todos los requisitos a lo largo de los programas para 
obtener su grado, no desarrollan o finalizan la tesis (Carlino, 2005). Lo anterior se debe a las dificultades que tienen para escribir ese género discursivo y a la falta de orientación en el proceso de elaboración de este. Esta situación puede tener consecuencias negativas para las instituciones de educación superior, pues si sus estudiantes de postgrado desertan, no se cumplen los criterios de calidad de los programas e, incluso, sus acreditaciones pueden peligrar. Por ende, surge la necesidad de crear iniciativas o dispositivos para acompañar, mediar, andamiar y retroalimentar el proceso de escritura de la tesis de postgrado. Especialmente, considerando que investigadoras como Natale (2014) han constatado la importancia de la retroalimentación en las devoluciones escritas por parte del profesor, durante el proceso de escritura del estudiante, para la calidad del rendimiento escrito.

Ciertamente, es en este contexto donde se inscribe el artículo Perfil del estudiante y desempeño en actividades virtuales de retroalimentación entre pares en torno a capitulos de tesis de posgrado de Hilda Difabio de Anglat y Guadalupe Álvarez, quienes implementaron un seminario de posgrado en modalidad virtual de 90 horas de duración en una facultad de humanidades de una universidad argentina. Este se llevó a cabo a través de la plataforma Moodle con documentos compartidos en Google Drive, donde pares y supervisores retrolimentaron los avances de escritura. Entre los resultados, se destaca que predominan los comentarios sobre el modelo textual con función directiva y desde una retroalimentación básica; sin embargo, las retroalimentaciones globales presentan más fundamentación. Asimismo, los estudiantes con perfiles inferior-intermedio en escritura académica ofrecen devoluciones más básicas, mientras que aquellos con perfiles intermedio-superior ofrecen comentarios con el doble de fundamentaciones. Con respecto a la evaluación de la experiencia por parte de los participantes, señalan su utilidad debido a que hay un foco en los detalles y que el uso de herramientas como Gogle Drive facilita la interacción y la actualización. En consecuencia, esta investigación puede contribuir a validar la evaluación entre pares en entornos virtuales como una experiencia positiva para el apredizaje de los estudiantes, pues desarrollan una mirada más crítica y reflexiva y desarrollan la deliveración sobre las dimensiones del género discursivo, el metalenguje y el contenido. Por último, esta investigación puede ofrecer luces respecto del desarrollo y seguimiento de los procesos de escritura académica en contextos de distanciamiento social por pandemias como la de la covid19.

Otra de las líneas de investigación sobre la escritura académica y disciplinar ha sido el estudio de las concepciones y representaciones tanto discursivas como sociales sobre la comunicación académica de distintos actores de la educación superior, como estudiantes y profesores, y en distintos ámbitos del conocimiento (Aguilar, Albarrán, Errázuriz \& Lagos, 2016; Errázuriz, 2017, 2020; Errázuriz \& Aguilar, 2020; Natale, 2011; Núñez \& Moreno-Núñez, 2017). En este sentido, el abordaje de las representaciones sociales sobre la escritura académica de los docentes resulta clave debido a que estas están vinculadas a las prácticas pedagógicas y a los aprendizajes del alumnado, es decir, pueden tener un impacto en las estrategias didácticas que despliega el profesorado y, por tanto, en el desempeño de sus estudiantes (Castorina \& Kaplan, 1997). En esta línea, podemos encontrar el artículo Representaciones sociales de docentes de una universidad tradicional chilena acerca de la escritura académica y de su enseñanza-aprendizaje de María Verónica Sánchez Gibbons. En él, la autora identifica y analiza las representaciones sociales de profesores de distintas facultades de una universidad chilena, lo que aporta información relevante dado que la mayoría de las investigaciones de este tipo se han centrado en los estudiantes; sin embargo, si deseamos realizar transformaciones del sistema es imperativo contar con más información sobre cómo se representan la escritura académica los formadores de profesionales.

Entre los resultados de este artículo, cabe señalar que se presentan como debilidades la ausencia del enfoque de escritura como proceso; la confusión entre la escritura académica, disciplinar y profesional y la creencia de que se puede aprender a escribir académicamente a través de la lectura de textos. Asimismo, se destacan como fortalezas la importancia que los docentes atribuyen a la evaluación y retroalimentación y a la práctica y motivación por parte de los estudiantes. Por otra parte, si bien los docentes adjudican una gran relevancia al desarrollo de la escritura académica en la universidad y que es necesario que se trabaje a través del currículo, presentan deficiencias en sus estrategias de enseñanza-aprendizaje en este ámbito, pues no atienden el proceso de escritura y muestran una confusión en cuanto a las características formales y estructurales de los géneros que se escriben en su comunidad disciplinar. En consecuencia, se vislumbra la necesidad de contar con lineamientos sobre alfabetización académica de orden intitucional, no solo a nivel de la universidad, sino que a nivel de cada facultad, así como también tener instancias de formación sobre la enseñanza- 
aprendizaje de la escritura académica en cada comunidad discursiva.

En relación con la línea de investigación desarrollada en torno a escribir para aprender o la escritura como herramienta epistémica, existen abundantes evidencias sobre cómo se usa la escritura como un dispositivo de aprendizaje en las distintas disciplinas. Es decir, los docentes aplican tareas de escritura y las evalúan para constatar el nivel de aprendizajes respecto de un contenido específico y también el nivel de reflexión sobre este. En este sentido, sabemos que mientras escribimos sobre un tema, leemos y averiguamos acerca de este, relacionamos y contrastamos los distintos puntos de vista, reflexionamos sobre lo escrito, creamos ideas y soluciones a partir de las reflexiones y, por tanto, aprendemos sobre el contenido del que escribimos (Klein, Boscolo, Kirkpatrick \& Gelati, 2014). Evidentemente, cuando escribimos, consideramos además otras dimensiones del fenómeno como comunicar a unos lectores determinados, lograr un propósito en los lectores o pertenecer a una comunidad. No obstante, el enfoque de escribir para aprender se centra en escribir para desarrollar aprendizajes sobre un conocimiento determinado (Bereiter \& Scardamalia, 1987; Carlino, 2005; Castelló, 2009). Es en esta perspectiva donde se adscribe el artículo Escritura colaborativa en linea. Un estudio de la revisión textual compartida en la formación inicial de maestros de María Begoña GómezDevís y Elia Saneleuterio, que corresponde a una investigación acción, mediante un proyecto de innovación, con el objetivo de analizar una experiencia de escritura colaborativa en línea.

Las autoras realizaron un seguimiento de un proceso de aprendizaje colaborativo que consisitió en la escritura de un diario virtual que recoge las sesiones de la asignatura Lengua Española para la formación de profesores. De este modo, 84 estudiantes moderaron una conversación virtual con una media de 12-16 mensajes de autoría múltiple. Algunas de las conclusiones más relevantes han sido comprobar que la mediación del profesor tiene impacto en la cantidad y calidad de los comentarios y que la coevaluación de pares promueve los procesos argumentativos y reflexivos y un mayor desempeño escrito. Asimismo, a través de esta modalidad es posible conformar comunidades de aprendizaje comprometidas, andamiar y acompañar a los participantes y, así, entregarles ayudas y retroalimentación durante el proceso. Asimismo, al igual que el artículo mencionado anteriormente de Difabio de Anglat y Guadalupe Álvarez, este trabajo puede contribuir a ofrecer soluciones y propuestas para desarrollar los procesos de escritura académica a distancia en contextos de confinamiento.

En los párrafos anteriores también se llama la atención sobre las dificultades de escritura que tienen los estudiantes universitarios. Al respecto, es común escuchar la crítica de los profesores de cualquier disciplina sobre lo mal que escriben sus estudiantes. Así, unos hacen referencia a la cantidad de faltas de ortografía que cometen y al valor que en la calificación final dan a este aspecto; otros señalan el desconocimiento de las normas de citación empleadas en la disciplina; otros también indican la ausencia de coherencia y cohesión de los textos, y de habilidades de lectura crítica o de conocimiento de estrategias argumentativas, entre otros comentarios. Tanto es así que incluso es frecuente leer en los medios de comunicación titulares sobre esta falta de competencia comunicativa de los estudiantes universitarios. Se sabe que estos, como se argumenta aquí, no tienen por qué dominar las convenciones discursivas de la comunidad académica en la que se encuentran cursando sus estudios superiores antes de comenzar la universidad. También se sabe que es cierto que ingresan con carencias vinculadas a la expresión escrita. Sin embargo, lo preocupante es la aparente falta de evolución y resolución de dificultades, incluso su aumento, durante sus estudios en algunas cuestiones, como dan cuenta Alba Naroa Romero y Manuela de las Nieves Álvarez en su trabajo Grado de alfabetización académica y prácticas escritoras en el marco de un programa formativo. Esto refuerza la idea de que el aprendizaje de la escritura es un proceso complejo que dura toda la vida, pero también la de que la universidad tiene que tomar medidas al respecto.

Una de las principales, como hacen las autoras, es atender a las dificultades de escritura de los estudiantes. En este sentido, no se considera que sobren las investigaciones sobre la cuestión si se parte de la premisa de que la enseñanza de la escritura debe ser una actividad situada en un contexto determinado. Así, conocer la realidad de cada centro y al comienzo de los estudios superiores es fundamental para adaptar, en función de las necesidades, planes de alfabetización académica graduales durante las carreras. Además, con relación a esto, como realizan Romero y Álvarez, es necesario conocer qué géneros discursivos se trabajan. Su trabajo muestra cómo prevalecen unos frente a otros durante los cuatro cursos de un grado universitario en España y cómo algunos de ellos son poco exigentes discursivamente. Inciden, asimismo, en la necesidad de una enseñanza gradual de los géneros para lo que sería necesaria la coordinación en los grados. Al respecto, cabe señalar que en el 
ámbito que ocupa el presente monográfico son prolíferas las investigaciones que tienen como objetivo caracterizar los géneros académicos. También estas siguen siendo pertinentes pues, si bien en el ámbito iberoamericano se comparte el idioma español y el portugués, en cada país, en cada universidad, en cada carrera se entiende por algunos géneros algo diferentes. ¿Qué es una monografía en España? ¿Y un trabajo escrito en Chile? ¿Qué se espera de un ensayo en cuarto curso de un grado de Pedagogía en los diferentes países? Investigaciones si cabe más necesarias ahora en un contexto de internacionalización y virtualización de la universidad que permite a estudiantes de un lugar formarse en instituciones superiores de otro.

Otras de las iniciativas de alfabetización académica que ocupa la atención de este monográfico son los centros de escritura. Estos, si bien surgieron en países anglosajones como EE.UU. o Reino Unido hace varias décadas, han proliferado en Latinoamérica y España una vez comenzado el siglo XXI. Cabe señalar que estos centros se crearon con la finalidad de atender a las dificultades de escritura y que, con el tiempo, bajo la premisa de hacer mejores escritores y no mejores escritos, adoptaron estrategias didácticas centradas en el proceso de escritura. De otra parte, los centros de escritura han sido criticados por tener como eje principal la atención individualizada a los estudiantes a través de tutorías de escritura y, en consecuencia, el parcial impacto que tienen en la mejora de la expresión escrita de todos los estudiantes de una institución dado que es muy complicado atender a todos. No obstante, esa atención individualizada ha de entenderse como condición en ocasiones imprescindible para alcanzar la calidad y equidad antes mencionada. Sea por las características personales de cada estudiante, sea por la ausencia de tutorización de algunos profesores en las disciplinas, sea porque no se han enseñado los géneros discursivos, entre otras razones.

Por otro lado, también ha existido cierta reticencia hacia los centros de escritura por conllevar un esfuerzo institucional que en función del contexto puede encontrarse con multitud de escollos, siendo el factor económico y la cultura institucional los principales. Pues bien, al margen de que detrás de esta idea haya una velada crítica al supuesto carácter elitista de los centros de escritura, son numerosos los ejemplos y las investigaciones que demuestran cómo los centros de escritura son sostenibles económicamente y ofrecen un servicio que redunda en la mejora de las destrezas comunicativas de los estudiantes y la calidad docente de los profesores. En este sentido, un modelo de centro de escritura adaptado a la actualidad ha de entenderse como un espacio institucional que facilita la oportunidad de promover la enseñanza de la escritura no solo a través de tutorías ni de talleres o recursos tan solo a disposición de los estudiantes. También lo hará a través de un diálogo continuo con gestores y profesores universitarios a fin de institucionalizar programas de enseñanza del proceso de escritura y los géneros académicos a través del currículum y de las disciplinas, y la consecuente formación del profesorado. Son un espacio, al fin y al cabo, en donde a través de la innovación educativa se puede gestar una reforma - de nuevo institucional- que implique una política de alfabetización académica. Son también, por tanto, una muestra evidente de cómo innovación y reforma constituyen las dos caras de una misma moneda.

Por todo ello, para conocerlos más de cerca y observar cómo la labor de un centro de escritura no se queda en la simple oferta de tutorías y puede trascender a asignaturas específicas en diferentes carreras siempre que haya voluntad de mejorar la atención a los estudiantes universitarios sugerimos la lectura del texto Una intervención formativa del Centro de Escritura Nebrija en la Facultad de Ciencias Sociales de Alicia de la Peña y Rocío Santamaría. En él, en el marco de la asignatura de Diseños de investigación en Psicología se da cuenta de cómo a través de diferentes talleres, del trabajo colaborativo y de las tutorías de escritura se puede enseñar un género discursivo en una asignatura.

Además del centro de escritura en sus distintas modalidades, como el referido previamente, existen otros dispositivos para desarrollar la comunicación académica y disciplinar en educación superior, así ha surgido el modelo de co-docencia entre un docente disciplinar y un profesor de lengua. Estas iniciativas se han implementado en Latinoamérica, en particular en Argentina con bastante éxito, y consisten en el trabajo colaborativo entre un profesor de lengua que asesora a uno de una disciplina específica. De este modo, el trabajo con la lectura y escritura disciplinar se lleva a cabo de manera situada e integrado en los contenidos de las asignaturas o cursos y a través del currículo de los programas de formación. Estas parejas pedagógicas pueden operar de distintas maneras como en: el diseño conjunto de recursos didácticos, actividades e instrumentos de evaluación; impartir clases en conjunto, evaluar tareas de escritura colaborativamente e investigar los géneros discursivos o fenómenos de lectura y escritura disciplinar (Coffin \& Donohue, 2014; Natale, 2013). El beneficio de este tipo de dispositivos radica en que promueven una transformación institucional progresiva al 
involucrar a docentes, facultades disciplinares y especialistas en lengua. Así, se desarrolla una pedagogía colaborativa de carácter interdisciplinar, intertextual y dialógico, cuyos efectos positivos también son valorados por los estudiantes (Natale \& Stagnaro, 2017). Este es el tipo de iniciativas que aborda el artículo La co-docencia para el abordaje de la alfabetización académica: efectos en la formación de los docentes disciplinares de Lucía Natale.

Esta investigación analiza los efectos del desarrollo del modelo de co-docencia en el marco del Programa de Apoyo a la Lectura y la Escritura a lo largo del Currículum (PRODEAC), un programa implementado en una universidad pública argentina. Para ello, se ha efectuado un estudio cualitativo, mediante la teoría fundamentada, a partir de quince entrevistas en profundidad a docentes disciplinares que participaron del programa. En particular, se abordaron dos dimensiones: la enseñanza de la escritura en las disciplinas y las transformaciones en la planificación de las asignaturas. Entre los resultados, se destaca que este dispositivo favorece la reflexión acerca de lo que significa e implica escribir en las disciplinas y la reorganización de los cursos. En efecto, los participantes declaran haber aprendido estrategias didácticas para incluir la escritura en los contenidos de la asignatura e integrar criterios para diseñar los instrumentos de evaluación que aplican. Asimismo, también señalan haber percibido la valoración de los cambios por parte de los estudiantes, quienes mostraron una mayor autoconfianza al escribir. Por último, a partir de lo anteriormente aseverado, es posible concluir que la co-docencia es un dispositivo efectivo para la formación de calidad de profesionales, la transformación de las dinámicas institucionales, el fomento de la interdisciplinariedad y la mayor inclusión e inserción de los estudiantes durante sus estudios.

Por todo lo puesto, se considera que los artículos que se presentan en este monográfico sobre alfabetización académica dan cuenta de, si no todas, muchas de las investigaciones e iniciativas sobre enseñanza de la escritura en la universidad que se están poniendo en marcha en la actualidad en el contexto iberoamericano. Se considera que, junto con otros aportes realizados, pueden ser documentos que impulsen o dinamicen la toma de conciencia y la puesta en práctica de acciones de alfabetización académica. Así, quienes suscriben la coordinación de este monográfico se guiaron bajo la premisa de que, pese a la heterogeneidad de contextos y realidades, compartir el español como idioma puede ser una oportunidad para socializar preocupaciones similares -que no idénticas- y experiencias de éxito de las que todos los interesados en la equidad y la calidad de la educación superior podemos aprender.

\section{Referencias}

Aguilar, P., Albarrán, P., Errázuriz, M. C., \& Lagos, C. (2016). Teorías implícitas sobre la escritura: Relación de las concepciones de estudiantes de Pedagogía Básica con la calidad de sus textos. Estudios Pedagógicos 42(3), 7-26. doi: 10.4067/S0718-07052016000400001

Bazerman, C., Dean, C., Early, J., Lunsford, K., Null, S., Rogers, P., \& Stansell, A. (Eds.). (2012). International advances in writing research: Cultures, places, measures, Perspectives on writing. Fort Collins, CO: The WAC Clearinghouse and Parlor Press.

Bazerman, C., Little, J., Bethel, L., Chavkin, T., Fouquette, D., \& Garufis, J. (2005). Reference guide to writing across the curriculum. Parlor Press LLC.

Bereiter, C., \& Scardamalia, M. (1987). The psychology of written composition. London: LawrenceErlbaum

Carlino, P. (2013). Alfabetización académica diez años después. Revista Mexicana de Investigación Educativa, 18 (57), 355-38.

Carlino, P. (2005). Escribir, leer y aprender en la universidad: una introducción a la alfabetización académica (p. 200). Buenos Aires: Fondo de Cultura Económica.

Carlino, P. (2005). ¿Por qué no se completan las tesis en los posgrados? Obstáculos percibidos por maestrandos en curso y magister exitosos. Educere, Revista Venezolana de Educación, 9(30), 415-420.

Cassany, D. (2006). Tras las lineas: Sobre la lectura contemporánea. Barcelona: Graó.

Castelló, M. (2009). Los efectos de los afectos en la comunicación académica. En M. CASTELLÓ (Ed.), Escribiry comunicarse en contextos científicos y académicos. Barcelona: Graó.

Castorina, J.A. \& Kaplan, C. (1997). Representaciones sociales y trayectorias educativas. Una relación problemática. Educaçao \& Realidade, 22(2), 187-202.

Coffin, C., \& Donohue, J. (2014). A language as social semiotic based approach to teaching and learning in 
higher education. Oxford: Wiley-Blackwell

Errázuriz, M. C. (2020). Teorías implícitas sobre escritura de estudiantes y formadores de pedagogía. Magis. Revista Internacional de Investigación en Educación, 13, 1-26. doi: 10.11144/Javeriana.m13.tiee

Errázuriz, M. C. (2019). Desempeño escrito de estudiantes de programas de formación inicial docente: ¿Cómo es la calidad del proceso de escritura de sus ensayos? Lengua y Habla, 23, 224-242. Recuperado de: https://erevistas.saber.ula.ve/index.php/lenguayhabla/article/view/15670

Errázuriz, M. C. (2017). Teorías implícitas sobre escritura académica de estudiantes de programas de formación inicial docente: ¿Inciden en el desempeño escrito? Signo y Pensamiento, 36(71), 34-50

Errázuriz, M. C. (2017). Las tutorías de un centro de escritura como dispositivo de modelamiento de estudiantes de programas de formación inicial docente. En Natale, L. \& Stagnaro D. (Eds.). Alfabetización académica: un camino para la inclusión en el nivel superior. Los Polvorines: UNGS.

Errázuriz, M. C. (2016). The academic writing development in the initial teacher training: Tutoring in a writing centre as a strategy for the modelling of students. The International Journal of Literacies, 23(3), 27-43.

Errázuriz, M. C., \& Aguilar, P. (2019). Reconstrucción discursiva de teorías implícitas sobre la escritura de estudiantes universitarios de educación primaria. Lenguas Modernas, 53, 9-30. Recuperado de: https://lenguasmodernas.uchile.cl/index.php/LM/article/view/55444

Ezcurra, A. M. (2012). Igualdad en Educación Superior. Un desafio mundial. Los Polvorines: Universidad Nacional de General Sarmiento.

Ivanic, R. (1998). Writing and Identity: the discoursal construction of identity in academic writing. Amsterdam: John Benjamins Publishing. doi: 10.1075/swll.5

Klein, P., Boscolo, P., Kirkpatrick, L., \& Gelati, C. (Eds.). (2014). Writing as a learning activity. Leiden: Brill. doi: 10.1163/9789004265011

Martin, E., \& Cervi, J. (2006). Modelos de formación docente para el cambio de concepciones de los profesores. En J. I. Pozo, N. Scheuer, N., M. Del Puy Pérez, M. De la Cruz, E. Martin \& M. Mateos, Nuevas formas de pensar la enseñanza y el aprendizaje. Las concepciones de profesores y alumnos. Barcelona: Graó.

Natale, L. (2014). Interrelaciones entre representaciones discursivas sobre la escritura académica y devoluciones escritas de docentes universitarios. Onomázein, IX ALSFAL, 81-98. doi: 10.7764/onomazein.alsfal.6

Natale, L. (2013). Integración de enfoques en un programa institucional para el desarrollo de la escritura académica y profesional. Revista Mexicana de Investigación, 58(XVIII), 685-707. Recuperado de http://ref.scielo.org/stt653

Natale, L. (2011). La enseñanza y el aprendizaje de los géneros discursivos en la educación superior. Interacciones entre las representaciones de los profesores universitarios sobre la escritura académica, las valoraciones acerca de los estudiantes ya la elección de los géneros solicitados en las materias. Texturas, 11(10), 87-109. doi: 10.14409/texturas.v1111.2900

Natale, L., \& Stagnaro, D. (Eds.). (2017). Alfabetización académica: un camino para la inclusión en el nivel superior. Los Polvorines: UNGS.

Núñez, J. A., \& Moreno-Núñez, A. (2017). Percepción de los estudiantes universitarios iberoamericanos sobre la competencia comunicativa y la alfabetización académica. Zona Próxima, 26, 44-60.

Parodi, G., Ibáñez, R., Venegas, R., \& González, C. (2010). Identificación de géneros académicos y géneros profesionales: principios teóricos y propuesta metodológica. En G. Parodi (Ed.), Alfabetización académica y profesional en el siglo XXI: Leer y escribir desde las disciplinas (pp. 249-289). Madrid: Ariel.

Rose, D., \& Martin, J. (2012). Reading to Learn, Learning to Write. Genre, Knowledge and Pedagogy in the Sydney School. London: Equinox.

Russell, D. (1990). Writing Across the Curriculum in Historical Perspective: Toward a Social Interpretation. College English, 52, 52-73. doi: 10.2307/377412

Thaiss, C., Bräuer, G., Carlino, P., Ganobcsik-Williams, L., \& Sinha, A. (Eds.). (2012). Writing Programs Worldwide: Profiles of Academic Writing in Many Places. Fort Collins, CO: The WAC Clearinghouse \& Parlor Press. 